\title{
ACYCLOVIR for SARS-CoV-2: An Old Drug with a New Therapeutic Purpose - An Observational Study
}

\section{Vina S. Baker MD, MPH\&TM}

The Baker Clinic, New Iberia, LA, USA.

Corresponding Author: Vina S. Baker, the Baker Clinic, New Iberia, LA, USA. ORCID: 0000-0002-5478-0351.

Received date: December 20, 2021; Accepted date: January 04, 2022; Published date: January 10, 2022

Citation: Vina S. Baker. (2022). ACYCLOVIR for SARS-CoV-2: An Old Drug with a New Therapeutic Purpose - An Observational Study. International Journal of Clinical Case Reports and Reviews. 10(2); DOI:10.31579/2690-4861/199

Copyright: @2022 Vina S. Baker, This is an open-access article distributed under the terms of The Creative Commons. Attribution License, which permits unrestricted use, distribution, and reproduction in any medium, provided the original author and source are credited.

\begin{abstract}
Background: Food and Drug Administration (FDA)-approved outpatient oral treatment for COVID-19 has been lacking. Currently approved antiviral treatment for hospitalized patients is remdesivir. Approved outpatient preventive treatment has been vaccination. Outpatient adjunct treatment has been intravenous monoclonal antibody (Ab) infusion which aids in the human body's immune response to the invasion from SARS-CoV-2 virus. The latter requires multiple steps for completion and does not provide complete effective antiviral treatment to resolve viral burden (clinical observation). The world needs an effective, safe, inexpensive oral antiviral treatment for SARS-CoV-2.
\end{abstract}

Materials and Methods: We conducted a prospective observational study of SARS-CoV-2 exposed individuals ranging from asymptomatic to critical states of illness. We monitored 65 individuals residing in the United States of America (USA) from exposure and diagnosis of SARS-CoV-2 to recovery from viral burden. Individuals were evaluated for establishment of diagnosis of COVID19 , assessed for severity of illness, initiated individualized treatment regimen, and followed at intervals as needed until recovery or death. Laboratory studies included comprehensive metabolic panel, complete blood count, c-reactive protein, erythrocyte sedimentation rate, d-dimer, SARS-CoV-2 PCR test, serologic test for SARS-CoV-2 IgM and IgG antibodies. Imaging studies included chest radiography and $\mathrm{CT}$ of chest as needed. Progressive follow up care is ongoing.

Results: 63 patients recovered from infection from SARS-CoV-2, one patient died due to multiple complications while inpatient. One patient was lost to follow up care. Initiation of acyclovir and adjunctive treatment as early as possible provided the best outcome in minimizing clinical symptoms and disease burden related to SARS-CoV-2 in patients ranging from asymptomatic to critical illness.

Conclusions: Acyclovir, as the primary antiviral agent, is effective, safe, and inexpensive in decreasing initial and potential longterm impacts from SARS-CoV-2 virus infection in humans. It has proved to be beneficial in preventing hospitalization for high-risk individuals with mild to critical illness.

Keywords: SARS-CoV-2; COVID-19; long; antiviral; post-acute condition; syndrome; sequelae

\section{Introduction}

The World Health Organization (WHO) declared COVID-19 outbreak a global pandemic in March 2020. As of November 2021, approximately five million deaths worldwide and 750,000 deaths in the United States have been recorded [1]. To date, we have yet to have an FDA approved oral agent for the treatment of COVID-19. COVID-19 due to SARS-CoV2 virus ranges from asymptomatic to critical illness and death [2]. As with many infectious illnesses, early intervention provides for the least disease burden and is vital in successful treatment of infections. SARS-CoV-2 would not be an exception.

More recently, it has been reported that patients suffer from long term symptoms related to SARS-CoV-2 infection [3]. The National Institutes of Health (NIH) and National Health Service (NHS) have acknowledged long term sequelae related to COVID-19 [3,4]. Different terms are being circulated from long COVID, post-COVID condition, post-COVID syndrome, post-acute sequelae of COVID-19 to chronic COVID syndrome. Studies are underway to determine causality and possible treatment options [5].

Early in the pandemic, there were no FDA guidelines for outpatient treatment of COVID-19. During this time, we utilized a previously well tolerated, inexpensive, easy to administer antiviral agent: acyclovir.

Acyclovir was developed in 1974.The FDA approved it as the first successful antiviral agent in 1982 for the treatment of herpes virus infections. It carries a category B classification for use in pregnancy [6]. Acyclovir has proven to be well tolerated by the full range of populations 
as it has become a primary treatment for herpes virus infections from newborns to the elderly [7].

We previously reported in case studies that acyclovir was beneficial for treatment of severe pulmonary disease with potential to acute respiratory distress syndrome and splenomegaly related to SARS-CoV-2 [8].

This observational study aimed to investigate utilizing acyclovir as the primary antiviral treatment against SARS-CoV-2 infection and its role in minimizing disease burden of COVID-19 among individuals with asymptomatic to critical illness.

\section{Materials and Methods:}

Study Population: A total of 65 patients who presented to an outpatient clinic in the USA from April 2020 to November 2021 that were diagnosed with COVID-19 were included in this study. 58 patients were diagnosed via nasal swab specimen with PCR or Rapid Ag tests commercially available. Seven patients were diagnosed after undergoing serologic testing with SARS-CoV-2 commercially available antibody test.

We initiated a treatment regimen at initial visit for COVID-19 regardless of confirmation diagnosis by PCR test or once a diagnosis for COVID-19 was established by serologic testing.

This group of 65 patients were followed from initial visit for COVID-19 to present. We utilized the NIH Clinical Spectrum of SARS-CoV-2 Infection Guideline to categorize severity of illness [3].
Diagnostic Testing: Chest Xray, Chest CT, chemistry panel which includes liver and renal function indices, complete blood count, Creactive protein (CRP), erythrocyte sedimentation rate (ESR), d-dimer. CRP and ESR were reported as multiples (X) of upper limit of normal range.

Follow Up Visits: Patients were followed on an as needed basis until recovery or death in an outpatient or inpatient setting. Patients were given a blank sheet to write down any symptoms or questions they had at each visit. Telemedicine visits were used during lockdown periods.

\section{Recovery Definition:}

1. Resolution of symptoms from initial infection

2. Resolution of acute radiologic findings

3. Improvement of inflammatory markers

4. Return to activities engaged in prior to infection

\section{RESULTS:}

\section{Patient Characteristics:}

55 out of 65 patients were aged 40 to 80 , with 34 of them aged 61 to 80 . 47 out of 65 patients $(72 \%)$ had one or more comorbidities which places them at high risk for progression to severe disease and need for hospitalization [3]. 36 out of 65 patients (55\%) were classified as moderate to severe illness with bordering on critical illness. Seven patients were categorized with critical illness. (Table 1)

\begin{tabular}{|c|c|}
\hline Characteristics & Patients $(\mathrm{N}=65)$ \\
\hline \multicolumn{2}{|l|}{ SEX } \\
\hline Male & 14 \\
\hline Female & 51 \\
\hline \multicolumn{2}{|l|}{ AGE } \\
\hline $21-30$ & 2 \\
\hline $31-40$ & 5 \\
\hline $41-50$ & 8 \\
\hline $51-60$ & 12 \\
\hline $61-70$ & 15 \\
\hline $71-80$ & 19 \\
\hline $81-90$ & 4 \\
\hline$>90$ & 0 \\
\hline \multicolumn{2}{|c|}{ CO-MORBID ILLNESSES PRESENT AT DIAGNOSIS } \\
\hline None of the below & 18 \\
\hline 1 of the below & 16 \\
\hline 2 of the below & 12 \\
\hline 3 or more of the below & 19 \\
\hline Cardiovascular disease, stroke & 9 \\
\hline Diabetes & 21 \\
\hline Cancer & 8 \\
\hline Chronic lung disease & 9 \\
\hline Kidney disease & 3 \\
\hline Hypertension & 33 \\
\hline Body mass index $>30$ & 27 \\
\hline \multicolumn{2}{|l|}{ ILLNESS SEVERITY } \\
\hline Asymptomatic-Mild & 1 \\
\hline Mild-Moderate & 21 \\
\hline Moderate-Severe & 23 \\
\hline Severe-Critical & 13 \\
\hline Critical & 7 \\
\hline
\end{tabular}

Table 1: Characteristics of Patients Treated with Acyclovir 


\section{Lab Results and Imaging Studies:}

Peak levels of c-reactive protein, erythrocyte sedimentation rate and ddimer are reported in Table 2 . Chemistry panel and complete blood counts were unremarkable except for one patient who presented with thrombocytopenia. Majority of patients had one or two inflammatory markers that were elevated. Imaging studies revealed 29 patients to have had some infiltrative process with 21 having bilateral disease. Chest CT was completed for six patients with one having bilateral, diffuse emboli and diffuse infiltrative disease (Table 3). One inpatient case had improvement of lung disease at day 12 by CT scan but did not survive due to multiple non-COVID complications.

\begin{tabular}{|c|c|}
\hline C-Reactive Protein (CRP) & $\mathrm{N}=65$ \\
\hline Normal & 22 \\
\hline $1.1 \mathrm{X}$ to $5 \mathrm{X}$ & 18 \\
\hline $5.1 \mathrm{X}$ to $10 \mathrm{X}$ & 8 \\
\hline $10.1 \mathrm{X}$ to $20 \mathrm{X}$ & 7 \\
\hline 21.1 to $30 \mathrm{X}$ & 2 \\
\hline$>30.1 X$ & 2 \\
\hline N/A & 6 \\
\hline $\begin{array}{c}\text { Erythrocyte Sedimentation } \\
\text { Rate (ESR) }\end{array}$ & $\mathrm{N}=65$ \\
\hline Normal & 32 \\
\hline $1.1 \mathrm{X}$ to $2 \mathrm{X}$ & 15 \\
\hline $2.1 X$ to $3 X$ & 7 \\
\hline $3.1 \mathrm{X}$ to $6 \mathrm{X}$ & 4 \\
\hline$>6.1 \mathrm{X}$ & 1 \\
\hline N/A & 6 \\
\hline D-dimer & $\mathrm{N}=65$ \\
\hline Normal & 26 \\
\hline $1.1 \mathrm{X}$ to $5 \mathrm{X}$ & 25 \\
\hline $5.1 \mathrm{X}$ to $10 \mathrm{X}$ & 5 \\
\hline $10.1 \mathrm{X}$ to $20 \mathrm{X}$ & 1 \\
\hline$>20 X$ & 2 \\
\hline N/A & 6 \\
\hline Chemistry Panel & No significant findings \\
\hline Complete Blood Count & $\begin{array}{l}1 \text { with thrombocytopenia at } \\
\mathrm{dx}\end{array}$ \\
\hline
\end{tabular}

Table 2: Laboratory Studies - Chem 12, CBC, ESR, CRP, D-dimer

\begin{tabular}{|c|c|}
\hline Chest Xray Findings & $\mathbf{N = 6 5}$ \\
\hline Normal & 24 \\
\hline Unilateral & 8 \\
\hline Bilateral & 21 \\
\hline N/A & 12 \\
\hline & \\
\hline & $\mathbf{N}=\mathbf{6}$ \\
\hline CT of Chest Findings & 6 \\
\hline Pulmonary Embolism & 1 \\
\hline
\end{tabular}

Table 3: Imaging Studies - CXR, Chest CT, CT of brain

\section{Clinical Course and Treatment:}

We started patients on treatment with acyclovir as early as possible (Table 4). They started a medication regimen on the day of initial visit or within 48 hours of exposure. One patient was started on acyclovir after 15 days of diagnosis due to need for inpatient care related to COVID where acyclovir was not utilized. Four patients had a delay in treatment after 31 days. One of them had a prolonged stay at a nursing home facility after hospitalization. Another two patients were diagnosed with COVID for workup of other conditions which triggered a delayed treatment. One patient had persistent symptoms more than one month after monoclonal $\mathrm{Ab}$ infusion treatment.
Seven patients started their treatment at an inpatient setting and were followed as outpatients once released to go home. One patient started treatment as an outpatient; however, they required inpatient care for progression of illness because of noncompliance with outpatient treatment (Table 4).

We administered a treatment regimen with varying durations until resolution of symptoms, improvement of inflammatory markers and resolution of radiologic findings (Table 5). 32 patients required treatment for 8 to 21 days. 15 patients required greater than 31 days due to diffuse disease and prolonged need for resolution of symptoms and radiologic 
findings. One patient was lost to follow up and thus not able to determine duration of treatment.

We prescribed adjunct medications as clinical presentations deemed necessary (Table 6). Oral inhaled bronchodilators and glucocorticoids were started for patients with symptoms of cough and dyspnea on exertion. Oral glucocorticoids were initiated for elevated inflammatory markers. Supplemental oxygen therapy was provided for six patients with four of them starting it as an outpatient when pulse oximetry oxygen saturation on room air $\left(\mathrm{SpO}_{2}\right)$ reading was $88 \%$ or lower. Two of them were weaned from oxygen that was initiated while in the hospital. Oral $\mathrm{H} 2$ blocker was initiated for 51 patients to allow for additional benefit to protect gastrointestinal tract from virus and prevent gastritis and peptic ulcer disease during illness. Oral and intravenous antibiotics were used as an adjunct therapy to prevent superimposed bacterial infections. 12 patients were treated with azithromycin which was initiated at an urgent care center prior to their first visit at our facility. 11 patients were treated with subcutaneous enoxaparin due to elevated d-dimer and elevated risk for embolism related to debility. One of the 11 patients had diffuse infiltrative process, deep venous thrombi, and diffuse pulmonary emboli with pulmonary infarct. This patient was able to avoid hospitalization with initiation of this treatment along with supplemental oxygen at home. Two patients received intravenous remdesivir while inpatient as hospital protocol suggested. 58 patients were unvaccinated. Ten patients received monoclonal antibody infusion treatment.

\begin{tabular}{|c|c|}
\hline Time to Start of Treatment with Acyclovir & $\mathbf{N = 6 5}$ \\
\hline$<48$ hours of first visit or diagnosis & 58 \\
\hline 2 to 7 days from first visit or diagnosis & 2 \\
\hline 8 to 14 days from first visit or diagnosis & 0 \\
\hline 15 to 30 days from first visit or diagnosis & 1 \\
\hline$>31$ days from first visit or diagnosis & 4 \\
\hline & \\
\hline Treatment Setting & $\mathbf{N = 6 5}$ \\
\hline Outpatient only & 58 \\
\hline Inpatient to Outpatient & 6 \\
\hline Outpatient to Inpatient & 1 \\
\hline
\end{tabular}

Table 4: Time to Treatment, Setting of Treatment

\begin{tabular}{|c|c|}
\hline $\begin{array}{c}\text { Duration of } \\
\text { Treatment with } \\
\text { Acyclovir }\end{array}$ & $\mathbf{N = 6 5}$ \\
\hline 7 days & 11 \\
\hline 8 to 14 days & 19 \\
\hline 15 to 21 days & 13 \\
\hline 22 to 30 days & 6 \\
\hline$>31$ days & 15 \\
\hline N/A & 1 \\
\hline
\end{tabular}

Table 5: Duration of Treatment with Acyclovir

\begin{tabular}{|c|c|}
\hline Additional Medications/Treatment & Number of patients \\
\hline Oral inhaled bronchodilators (ipratropium) & 21 \\
\hline Oral inhaled glucocorticoids (budesonide, fluticasone, mometasone) & $12,19,1$ \\
\hline Oral glucocorticoids (dexamethasone, prednisone, medroxyprogesterone) & $41,5,1$ \\
\hline Oral H2 Blocker (famotidine) & 51 \\
\hline Oral Antibiotics (amoxicillin, azithromycin, ciprofloxacin, levofloxacin) & $2,12,2,3$ \\
\hline Intravenous antibiotics (ceftriaxone, vancomycin) & 4,1 \\
\hline Supplemental Oxygen & 6 \\
\hline Subcutaneous Anticoagulation (enoxaparin) & 11 \\
\hline Remdesivir & 2 \\
\hline Monoclonal Ab infusion & 10 \\
\hline Vaccination (fully vaccinated, partially vaccinated, unvaccinated) & $4,3,58$ \\
\hline
\end{tabular}

Table 6: Adjunct Medications/Treatments

\section{Outcome:}

We followed patients from initial visit to present. 63 patients have been followed for more than three months. 37 patients have been followed for more than nine months (Table 7).

60 patients have recovered with no residual symptoms. Three patients have persistent symptoms related to their COVID illness. Two patients have symptoms of dysosmia and or dysgeusia at 15 months. One patient has symptoms of imbalance and lower extremity weakness despite continued physical therapy and rehabilitation at 12 months. One patient was transferred to a long-term care facility due to the patient's needs being greater than could be met at home and subsequently was lost to follow up. One patient died due to unvaccinated state, noncompliance with outpatient regimen requiring inpatient care with subsequent multiple inpatient complications including failure to liberate from sedation despite improvement of pulmonary findings by CT of chest (Table 8).

39 patients met the recovery definition within 30 days. Only one patient reached recovery after 90 days. 23 patients achieved recovery within 90 days but greater than 30 days (Table 9). 


\begin{tabular}{|c|c|}
\hline Duration (months) & $\mathbf{N = 6 3}$ \\
\hline$<3$ & 0 \\
\hline 3 to 6 & 14 \\
\hline 6 to 9 & 2 \\
\hline 9 to 12 & 18 \\
\hline 12 to 15 & 22 \\
\hline 15 to 18 & 6 \\
\hline$>18$ & 1 \\
\hline
\end{tabular}

Table 7: Duration of follow up

\begin{tabular}{|l|l|}
\hline Patient Outcomes & $\mathbf{N}=\mathbf{6 5}$ \\
\hline Recovered with NO prolonged symptoms & 60 \\
\hline Recovered with prolonged symptoms & 3 \\
\hline Reinfection & 0 \\
\hline Lost to follow up & 1 \\
\hline Deceased & 1 \\
\hline $\begin{array}{l}\text { Persistent Symptoms related to COVID-19 for greater } \\
\text { than 6 months }\end{array}$ & $\mathbf{N}=\mathbf{3}$ \\
\hline Fatigue & 0 \\
\hline Shortness of breath, dyspnea & 0 \\
\hline Dysosmia, dysgeusia & 2 \\
\hline Imbalance, weakness & 1 \\
\hline
\end{tabular}

Table 8: Outcomes

\begin{tabular}{|l|l|}
\hline Time to Recovery & $\mathrm{N}=64$ \\
\hline$<30$ days & 39 \\
\hline 31 to 60 days & 15 \\
\hline 61 to 90 days & 8 \\
\hline$>91$ days & 1 \\
\hline Lost to follow up & 1 \\
\hline
\end{tabular}

Table 9: Recovery Time

\section{Discussion:}

This observational study focused on using an old antiviral medication, acyclovir, for treatment of SARS-CoV-2 infection for the primary outcome of recovery from illness and secondary outcome of prevention of hospitalization. This cohort of established patients were at very high risk for progression to severe disease related to SARS-CoV-2 as 58\% were of age greater than 60 and $72 \%$ had one or more comorbid illnesses.

We set a standard to define recovery from acute illness. This resulted in a low incidence of prolonged symptoms related to COVID-19. $97 \%$ of our patients achieved full recovery as defined by resolution of acute symptoms, resolution of acute radiologic findings and improvement in inflammatory markers. Two of the 65 or $3.1 \%$ of patients had mild symptoms of dysgeusia or dysosmia at 12 months. Severity of dysgeusia or dysosmia was minimal with one patient reporting improvement to $90 \%$ of pre-COVID state and one patient reporting improvement to 60-75\%. One patient who had a prolonged illness at a nursing home after initial hospitalization had hearing loss, ataxia, and weakness causing dependence of another individual for all activities of daily living at our initial visit. At 6 months from exposure to SARS-CoV-2, hearing loss had resolved. At 12 months, the patient was able to ambulate with a walker for 50 feet with standby assistance and able to perform activities of daily living after outpatient rehabilitation.

Starting acyclovir and adjunct treatment as early as possible was vital in avoiding hospitalization. We started treatment at our first visit if the presentation was for evaluation for exposure to SARS-CoV-2 despite the time delay in obtaining confirmation of diagnosis by laboratory studies and radiologic studies. Six patients started their care in a hospital with five of them reaching recovery. The sixth patient has completed outpatient rehabilitation at 12 months as mentioned above.

Neutralizing monoclonal antibody treatment has been shown to reduce hospitalization for mild to moderate illness due to SARS-CoV-2 [9]. Disappointingly, it does not allow for complete recovery from illness and may pose a problem with emerging variants evading its efficacy [10]. Among the ten patients who underwent monoclonal antibody infusion in this study, five developed infiltrative disease by chest radiograph. Acyclovir with adjunct treatment allowed for successful recovery and prevented progression to severe disease or need for hospitalization.

$89 \%$ of patients were unvaccinated. Acyclovir proved to be effective in resolving illness from SARS-Cov-2 infection among those who were unvaccinated.

One patient who was unvaccinated, declined monoclonal antibody infusion and noncompliant with outpatient treatment died due to multiple complications including mechanical ventilation and severe sedation. This patient had improvement in pulmonary infiltrates according to chest CT at day 12; however, we were unsuccessful in weaning the sedation.

One patient developed bilateral deep venous thrombi and diffuse bilateral pulmonary emboli with pulmonary infarct. This patient was started on acyclovir, famotidine, oral inhaled bronchodilator, oral inhaled steroid, supplemental home oxygen therapy for $\mathrm{SpO}_{2}$ of $83 \%$ at room air and full dose subcutaneous enoxaparin. Patient was offered inpatient treatment, but the patient declined. This patient achieved near resolution of pulmonary emboli at 2 months and near resolution of diffuse infiltrates with pulmonary infarct at 2 months follow up chest CT (Figure 1). 

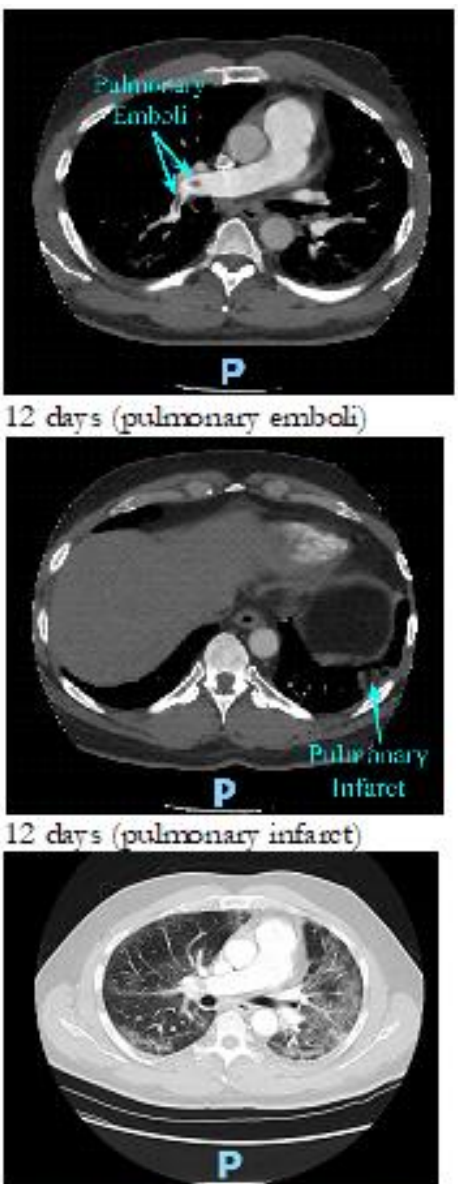

12 days (pulmonary infiltrates)

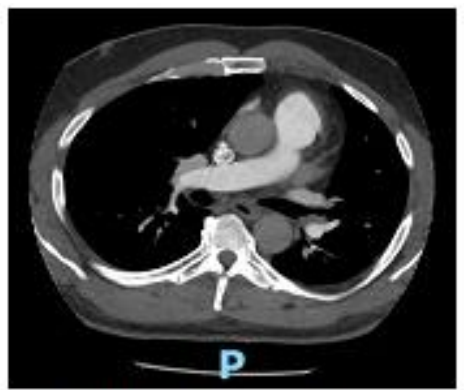

1 month (pulmonary emboli)

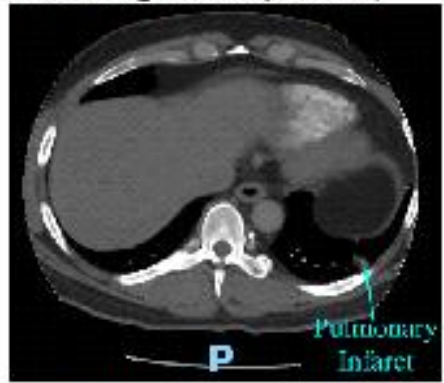

1 month (pulmonary infanct)

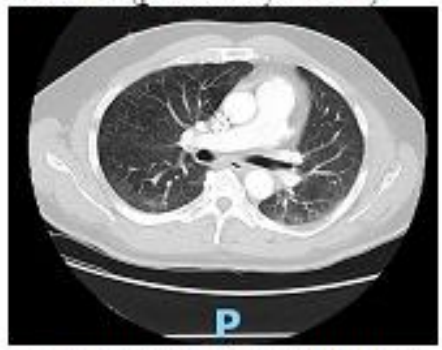

1 month (pulmonary infiltrates)

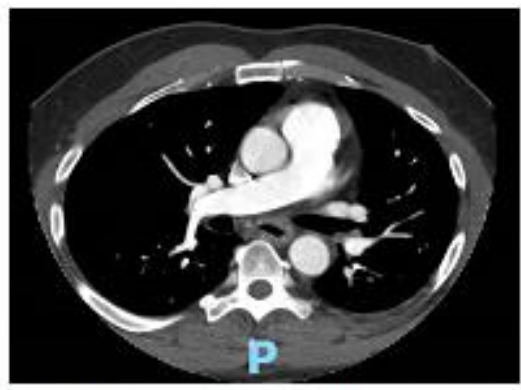

2 months (ptulmonary emboli)

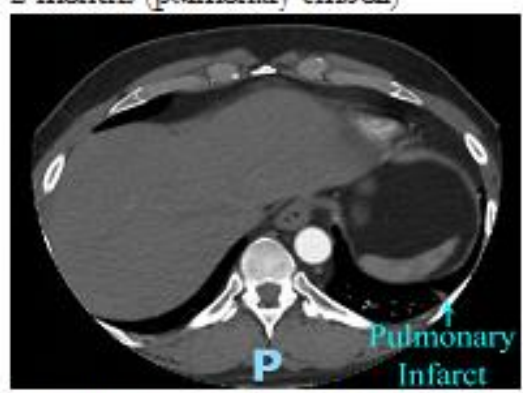

2 months (ptrlmonary infarct)

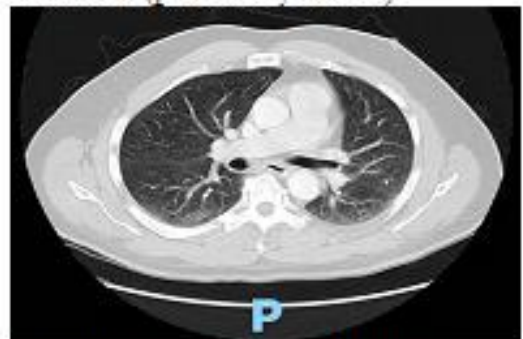

2 months (pulmonary infiltrates)

Figure 1: Chest CT Images 12 days, 1 month and 2 months from diagnosis

Limitations of this study revolve around the small number of cases. Symptoms prior to diagnosis or initial visit varied due to some patients seeking care at urgent care or emergency room prior to presentation to our outpatient facility. Recovery times may have been prolonged due to logistics of obtaining follow-up chest radiograph results, lab studies and scheduling of follow-up visit.

We have used acyclovir for our patients with excellent outcomes with minimal disease burden as evident by only three individuals with long term symptoms related to COVID 19 illness at 12 months. Individualizing treatment regimens for each patient with acyclovir as the primary agent has proved to be very effective, safe, and inexpensive to administer. Family support was vital in recovery of patients needing home oxygen therapy. Educating patients and their families regarding upright positioning for the initial phase of illness proved beneficial and assisted in avoiding the need for hospitalization. We propose the following as a starting point for approaching treatment for COVID 19 and its varied clinical presentations.

\section{SUGGESTED TREATMENT RECOMMENDATION:}

Exposure to Mild Symptoms: CYCLOPACK*: Acyclovir $400 \mathrm{mg}$ orally bid for 5 to 7 days, +/- H2 blocker (i.e., famotidine 20 mg daily for 5 to 7 days)

Mild to Moderate Symptoms: CYCLOPACKFLO*: Acyclovir 400 to $800 \mathrm{mg}$ orally bid for 7-10 days + oral steroid inhaler twice a day for 7 to 10 days $+\mathrm{H} 2$ blocker

Moderate to Severe Symptoms: CYCLOPACKPRO*: Acyclovir 400 to $800 \mathrm{mg}$ orally bid to tid + inhaled steroid twice a day for duration of symptoms, + oral anticholinergic bronchodilator (i.e, ipratropium, tiotropium, etc.) + $\mathrm{H} 2$ blocker + low dose oral steroid therapy + outpatient supplemental oxygen at $\mathrm{SpO}_{2}<92$

Severe to Critical Illness: CYCLOPACKMAX*: Acyclovir intravenously at $500 \mathrm{mg}$ daily to tid or 400 to $800 \mathrm{mg}$ orally bid to tid depending on renal function + oral inhaled steroid bid to tid + oral inhaled anticholinergic bronchodilator tid to qid + low dose oral steroids $+\mathrm{H} 2$ blocker + supplemental oxygen therapy when at $\mathrm{SpO}_{2}<94$, upright position, subcutaneous anticoagulation, selective serotonin reuptake inhibitor (SSRI) for mood symptoms, intravenous anti-bacterial agents and other treatments as needed

*CYCLOPACK - term used to describe combination of therapeutics in conjunction with acyclovir for treatment of COVID 19 


\section{CLINICAL PEARLS:}

The above suggestions SHOULD be altered for individualized therapy.

AVOID SEDATION as much as possible.

AVOID Beta Adrenergic Inhaled Agents unless absolutely necessary or patient was previously using it!

Maintain upright posture during early illness - avoid supine position as much as possible

Engagement of family is INVALUABLE

\section{Conclusion:}

Nearly two years into this pandemic, we are still struggling with the burden of disease related to SARS-CoV-2. We are fortunate to have effective vaccinations for prevention of severe illness. Somehow, this virus is proving to be more creative than our treatment efforts as new variants emerge. Initiation of acyclovir with adjunct treatment at time of diagnosis or within 48 hours of exposure provides a safe, effective, and inexpensive treatment for mild to critical illness in reaching recovery from SARS-CoV-2 infection and avoiding hospitalization. Further studies representing different parts of the world are warranted to validate effectiveness of acyclovir for SARS-CoV-2 infection among varied populations.

\section{References:}

1. World Health Organization. (2021). WHO Coronavirus (COVID-19) Dashboard. WHO Health Emergency Dashboard.

2. National Institute of Health. (2020). Therapies. NIH COVID19 Treatment Guideline.

3. National Institute of Health. (2020). Clinical Spectrum of SARS-CoV-2 Infection. NIH COVID-19 Treatment Guideline.

4. National Institute for Health and Care Excellence. (2021). COVID-19 Rapid Guideline: Managing the Long-Term Effects of COVID-19. NICE Guideline.
5. National Institute for Health. (2021). NIH Strategic Response to COVID-19. NIH COVID-19 Research.

6. Finch, Roger G. Acyclovir (Zovirax). eMedExpert.

7. Medscape. Zovirax (acyclovir) dosing, indications, interactions, adverse effects, and more.

8. Baker, Vina S. (2021). Acyclovir for SARS-CoV-2: An Old Drug with a New Purpose. Clinical Practice. 18(1);1584-1592.

9. Verderese JP, Stepanova M, Lam B, Racila A, Kolacevski A, Allen D, Hodson E, Aslani-Amoli B, Homeyer M, Stanmyre S, Stevens H, Garofalo S, Henry L, Venkatesan C, Gerber LH, Motew S, Jones JS, Younossi ZM. (2021). Neutralizing Monoclonal Antibody Treatment Reduces Hospitalization for Mild and Moderate COVID-19: A Real-World Experience. Clin Infect Dis.

10. Bariola JR, McCreary EK, Wadas RJ, Kip KE, Marroquin OC, Minnier T, Koscumb S, Collins K, Schmidhofer M, Shovel JA, Wisniewski MK, Sullivan C, Yealy DM, Nace DA, Huang DT, Haidar G, Khadem T, Linstrum K, Seymour CW, Montgomery SK, Angus DC, Snyder GM. (2021). Impact of Bamlanivimab Monoclonal Antibody Treatment on Hospitalization and Mortality Among Nonhospitalized Adults With Severe Acute Respiratory Syndrome Coronavirus 2 Infection. Open Forum Infect Dis. 8(7):ofab254.
This work is licensed under Creative Commons Attribution 4.0 License

\section{Submit Manuscript}

To Submit Your Article Click Here:

DOI: $10.31579 / 2690-4861 / 199$
Ready to submit your research? Choose Auctores and benefit from:

$>$ fast, convenient online submission

$>$ rigorous peer review by experienced research in your field

$>$ rapid publication on acceptance

$>$ authors retain copyrights

$>$ unique DOI for all articles

$>$ immediate, unrestricted online access

At Auctores, research is always in progress.

Learn more https://auctoresonline.org/journals/international-journal-ofclinical-case-reports-and-reviews 